\title{
Republished: Real-time MRI guidance for intra- arterial drug delivery in a patient with a brain tumor: technical note
}

\author{
Michal Zawadzki, ${ }^{1}$ Jerzy Walecki, ${ }^{1}$ Boguslaw Kostkiewicz, ${ }^{2}$ Kacper Kostyra, ${ }^{2}$ \\ Monica Smith Pearl, ${ }^{\text {3,4 }}$ Meiyappan Solaiyappan, ${ }^{3}$ Piotr Walczak, ${ }^{3}$ Miroslaw Janowski ${ }^{3}$
}

${ }^{1}$ Department of Radiology, Centre of Postgraduate Medical Education, Warsaw, Poland ${ }^{2}$ Department of Neurosurgery, Central Clinical Hospital of Ministry of the Interior and Administration, Warsaw, Poland ${ }^{3}$ Department of Radiology, Johns Hopkins Hospital, Baltimore, Maryland, USA ${ }^{4}$ Department of Radiology, Children's National Medical Center, Washington, District of Columbia, USA

\section{Correspondence to} Dr Miroslaw Janowski, Department of Radiology, Johns Hopkins University, Baltimore MD 21205, USA; mjanows1@ jhmi.edu

Republished with permission from BMJ Case Reports Published 27 January 2019; doi: 10.1136/bcr-2018-014469

Received 1 October 2018 Revised 19 November 2018 Accepted 19 November 2018 Published Online First 23 May 2019

Check for updates

(C) Author(s) (or their employer(s)) 2019. No commercial re-use. See rights and permissions. Published by BMJ.

To cite: Zawadzki M Walecki J, Kostkiewicz B, et al. J Neurolntervent Surg 2019;11:e3

\section{ABSTRACT}

Patients suffering from malignant brain tumors are burdened with a grim prognosis. The blood brain barrier is considered a primary obstacle in therapeutic drug delivery to the brain. Intra-arterial (IA) delivery of therapeutic agents following osmotic BBB opening has been attempted for years, but high variability has limited its widespread implementation. It has been recently shown in animal studies that MRI is superior to X-ray for guiding IA infusions, as it allows direct visualization of the brain parenchyma perfused and facilitates predictable drug targeting. Moreover, PET imaging has revealed that IA, not intravenous, delivery of bevacizumab results in brain accumulation, providing strong rationale for utilizing the IA route. Here, we present our experience in a patient with recurrent butterfly glioblastoma enrolled in first-in-man MRI-guided neurointervention for targeted IA drug delivery.

\section{BACKGROUND}

The blood-brain barrier (BBB) continues to be an obstacle to the effective delivery of therapeutic agents to the brain. Intra-arterial (IA) infusion of therapeutic agents following osmotic blood-brain barrier opening (OBBBO) under X-ray angiography guidance has been attempted for years, ${ }^{1}$ but it has not become a routine treatment partially due to the variability of outcomes. Interestingly, recent animal studies have shown an advantage of adding MRI to guide IA infusion instead of solely relying on $\mathrm{X}$-ray. ${ }^{2}$ Due to the low sensitivity of X-ray contrast agents, angiography requires a rapid bolus infusion of contrast and visualization of the smallest intracranial vessels is limited. MRI contrast agents, on the other hand, are highly sensitive and can detect the smallest concentrations of contrast, specifically at the microcirculation level. This inherent feature highlights the utility of MRI guidance and makes it possible to show the territory of the brain parenchyma supplied by the catheter, which is highly dynamic and variable. Importantly, both the infusion rate and catheter tip position can be modified to optimise the desired brain territory under MRI guidance.

IA delivery of bevacizumab after OBBBO based on X-ray angiography has been shown to be safe ${ }^{3}$ and at least as effective as intravenous administration with fewer systemic adverse effects. ${ }^{4}$ In one patient, IA bevacizumab was successful in treating two consecutive glioblastoma recurrences leading to long-term survival. ${ }^{5}$ Positron emission tomography (PET) imaging of radiolabeled bevacizumab infused intra-arterially in a mouse model unequivocally revealed superiority of IA delivery to the brain. OBBBO further potentiated drug uptake, while intravenously administered bevacizumab did not enter the brain at all, even after OBBBO. ${ }^{6}$

The IA route for delivery of therapeutic agents following OBBBO should be considered and further explored to optimise targeted drug delivery to the brain. This is a reasonable strategy to prolong the survival of patients with glioblastoma for whom no other therapeutic options exist. MRI guidance may provide an advantage over X-ray alone, and assists in the selection of arteries used for infusion of therapeutic agents.

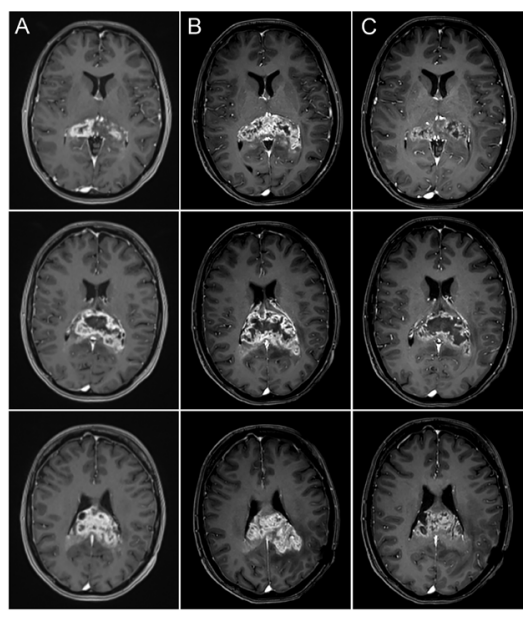

Figure 1 Axial T1 post-gadolinium weighted images of the brain before and after intra-arterial (IA) treatment show a tumor response. Corresponding axial slices of the brain 15 days (column A) and 4 days (column B) before and 3 days after IA blood brain barrier disruption (BBBD) and bevacizumab infusion (column $\mathrm{C}$ ) demonstrate rapid growth of the enhancing mass before treatment. After the procedure the tumor mass decreased in size as well as the degree of enhancement and mass effect. Reduction of edema after treatment can be concluded from subsiding $\mathrm{T} 1$ hypointensity around the tumor, which is evident when comparing columns $\mathrm{C}$ and $\mathrm{B}$. 


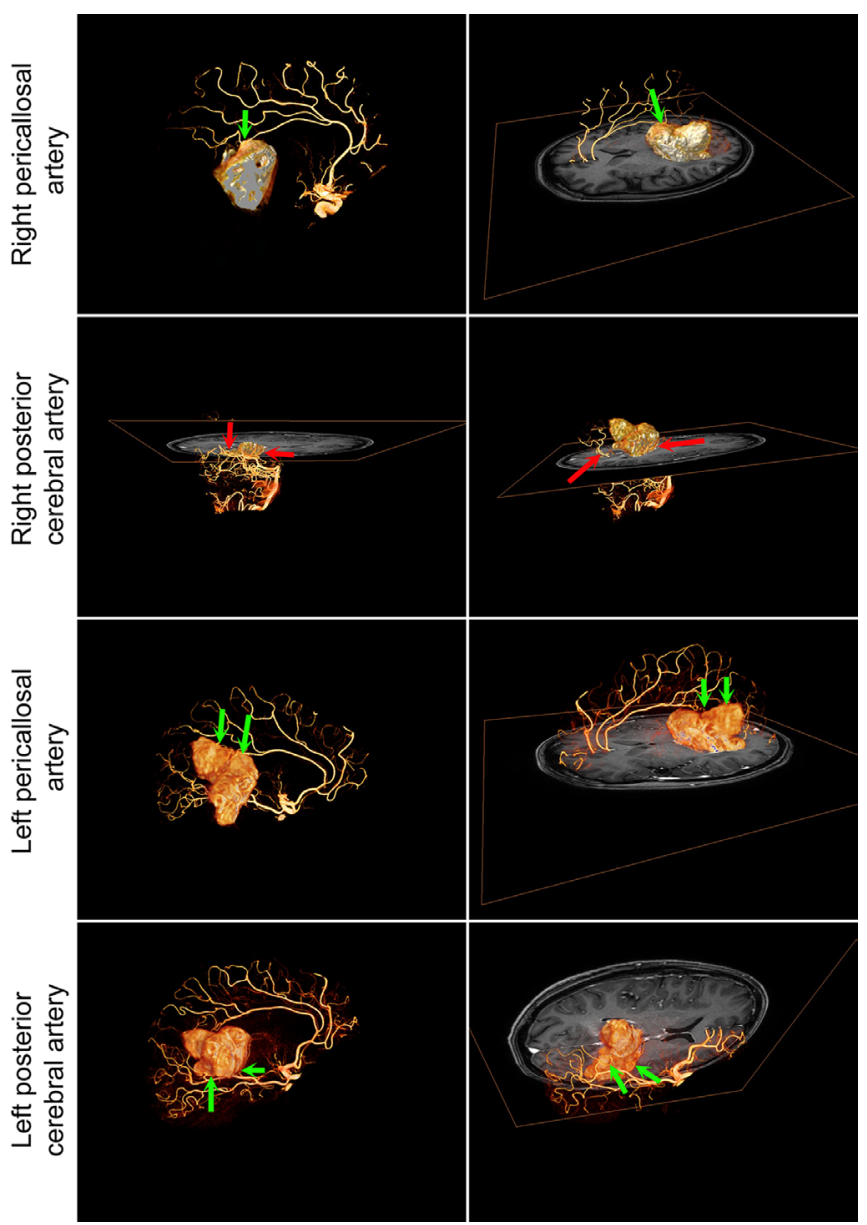

Figure 2 Four-vessel angiogram performed for planning purposes superimposed over MRI with segmented tumor. The green arrows indicate the vessels which appear to enter the tumor while the red arrows indicate vessels in the near vicinity of the tumor but without clear entry points. The figure was prepared using Amira software.

\section{CASE PRESENTATION}

A middle-aged adult patient with a history of left parietal glioblastoma presented with rapidly progressive cognitive decline. An MRI was performed which revealed a $41.15 \mathrm{~cm}^{3}$ enhancing mass consistent with recurrent butterfly glioblastoma (figure 1A). All standard conventional therapeutic options were exhausted, and treatment with IA bevacizumab was selected after approval from the institutional ethical committee. Bevacizumab was chosen because of the promising published results and also because of its advantageous safety profile. Specifically, compared with other experimental therapeutics such as immunotherapy or local chemotherapy, bevacizumab is the least likely to generate a potentially life-threating edematous response. This was a key factor in the choice of therapeutic agent for our patient, whose tumor was deeply seated in a highly sensitive brain region. Before the procedure the patient was walking independently, without motor or sensory deficits, with signs of cognitive deterioration as illustrated by the Mini-Mental State Examination (MMSE) score of 19 and Karnofsky Performance Status (KPS) of $60 \%$. A repeat MRI was performed 11 days later for pre-procedure planning and demonstrated a $25 \%$ increase in the size of the enhancing mass, now with a volume of $52.06 \mathrm{~cm}^{3}$ (figure $1 \mathrm{~B}$ ).

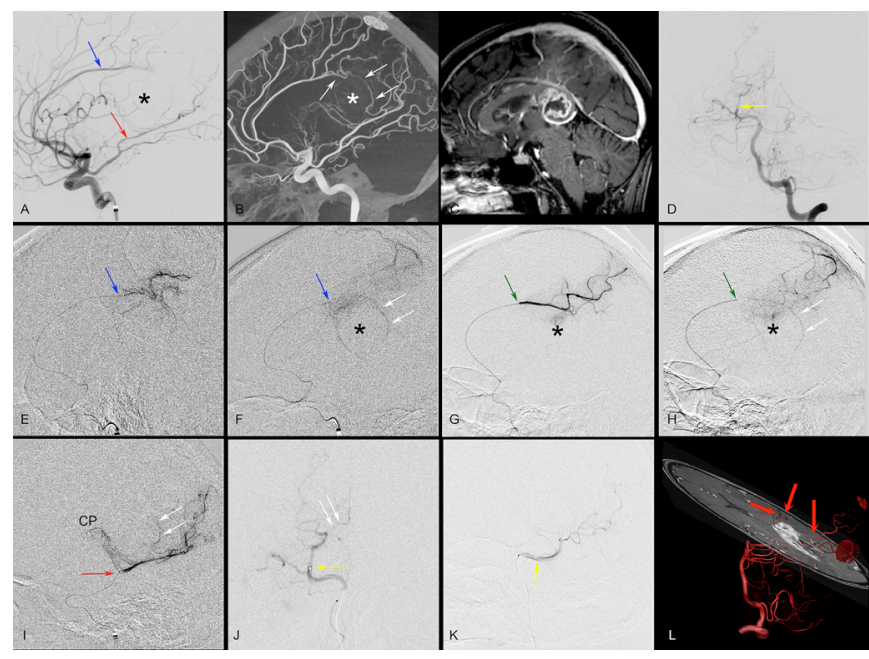

Figure 3 Fluoroscopic and digital subtraction angiography (DSA)guided drug delivery. Diagnostic cerebral DSA (A, D), 3D DSA (B), and sagittal T1 post-gadolinium $(C)$ images were obtained prior to intraarterial drug delivery. Lateral views from the left internal carotid artery (ICA) injection (A, B) show the pericallosal contributors (white arrows in $B$ ) to the splenial region glioblastoma, which has a faint tumoral blush (asterisk). The blue arrow (A) corresponds to the subsequent microcatheter position $(E, F)$ in the left pericallosal artery. Green arrows $(G, H)$ are similar, noting the microcatheter position in the right pericallosal artery. Note the minimal tumor blush (asterisk) with the microcatheter injections. The tumor borders can be faintly seen (F) around the asterisk. The left posterior cerebral artery (PCA) was accessed from the left ICA, as a prominent posterior communicating artery provided the left PCA territory (A, D; posteroanterior view of left vertebral artery angiogram). The red arrow in (A) shows the microcatheter position in the left PCA (I), which opacifies the choroid plexus (CP) and only faintly shows the posterior pericallosal/splenial contributors (white arrows). No distinct tumor blush is noted. Similarly, on the right parieto-occipital artery, microcatheter injections (yellow arrow in J, posteroanterior view; K, lateral view; D) no distinct tumor blush is noted. Even with the superimposition of 3D DSA and MRI, the distinct arterial supply to the tumor from the right PCA remained unclear. The red arrows $(\mathrm{L})$ point to vessels branching off the right PCA and surrounding tumor.

\section{INVESTIGATIONS}

Diagnostic cerebral angiography was performed and 3D digital subtraction angiography (DSA) images were overlaid on the MRI (figure 2). The image overlay suggested that four vessels contributed to the arterial supply of the tumor: the distal pericallosal branches of the bilateral anterior cerebral arteries (ACAs) and the splenial (posterior pericallosal) branches from the bilateral posterior cerebral arteries (PCAs). A hypoplastic left P1 segment was incidentally noted and a prominent left posterior communicating artery provided the left PCA territory. Despite the anatomical superimposition of 3D DSA images from the diagnostic angiogram over the MR-depicted tumor area, the precise contribution from each of the respective arterial feeders was unclear. The right PCA supply was the most ambiguous, even with a selective microcatheter injection on DSA, which failed to show the individual branches supplying the tumor or a distinct tumor blush (figure 3). Thus, real-time MRI was planned to better delineate the arterial contribution to the tumor from the right PCA, given the ambiguity on conventional DSA imaging. 


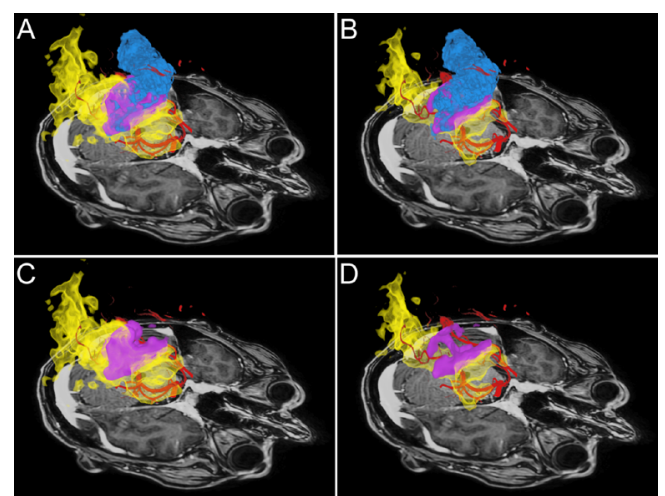

Figure 4 MRI-guided drug delivery: comparison between faster infusion $(1.5 \mathrm{~mL} / \mathrm{min})(A, C)$ and slower rate $(0.75 \mathrm{~mL} / \mathrm{min})(B, D)$. The upper row shows the overlap of tumor and intra-arterial infusion in purple. The tumor area non-perfused by the right posterior cerebral artery (PCA) is shown in blue while the territory supplied by the right PCA outside the tumor is shown in yellow. In the lower row the tumor volume has been removed to better emphasize the differences in the territory supplied by the catheter at a faster speed (C) and slower speed (D). The figure was prepared using Dextroscope Virtual Reality environment.

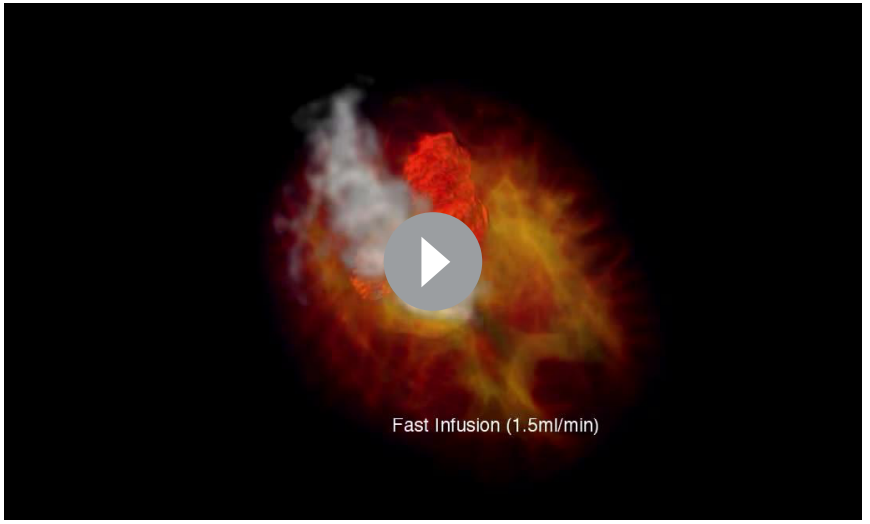

Video 1

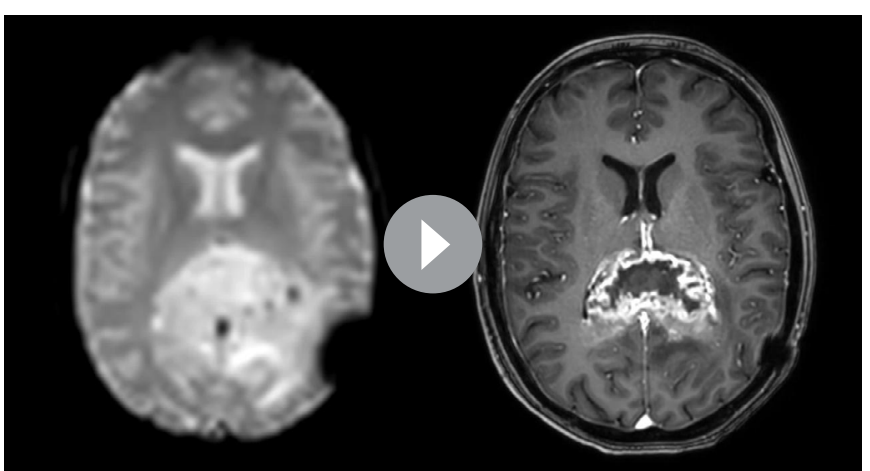

Video 2

\section{TREATMENT}

A $5 \mathrm{~F}$ guide catheter (5F 100VERT; Balton) and microcatheter (Magic 1.2F, Balt) were used to catheterize the bilateral pericallosal arteries and left PCA via right femoral arterial access. After mannitol-mediated BBB opening, bevacizumab solution (1:1 dilution in saline) was infused in each artery at a rate extrapolated from a previously published procedure. ${ }^{5}$

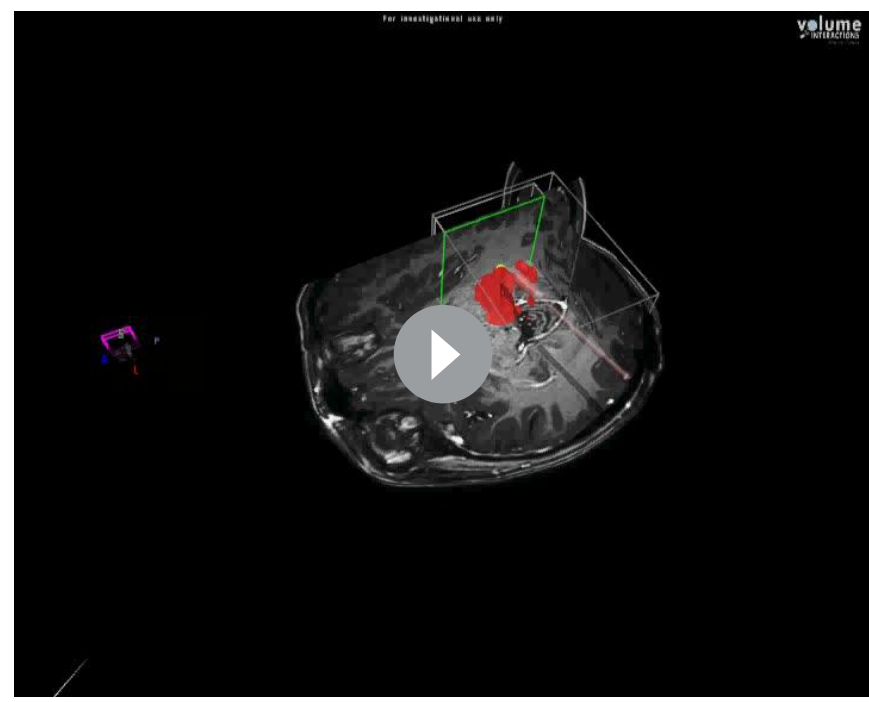

Video 3

The guide catheter was then placed in the distal V2 segment of the left vertebral artery and the Magic $1.2 \mathrm{~F}$ microcatheter was advanced into the right P3 segment, secured in place, and maintained on heparinized saline flush (5000 IU/L). Both catheters were non-ferromagnetic. As previously noted, no distinct tumorous blush from the right PCA was observed on DSA. The patient was transferred to the adjacent MRI suite (Phillips Ingenia 3T MR system) using an MR-compatible trolley. Real-time MRI using a standard gradient echo EPI pulse sequence of tumor volume and its margins (slab thickness $68 \mathrm{~mm}$ ) was performed. Two sequential microcatheter injections in the right parieto-occipital branch of the right PCA under MRI with gadolinium (ProHance 1:20 dilution in saline) were performed with differing speeds to assess potential areas of biodistribution. The first injection was performed at a rate of $1.5 \mathrm{~mL} / \mathrm{min}$. This infusion covered a volume of $117 \mathrm{~cm}^{3}$, of which $27 \mathrm{~cm}^{3}$ overlapped with the enhancing mass (figure 4). The second injection was performed at a rate of $0.75 \mathrm{~mL} / \mathrm{min}$ and covered a smaller volume of $67 \mathrm{~cm}^{3}$, of which $19 \mathrm{~cm}^{3}$ overlapped with the enhancing mass (see videos 1-3). Real-time MRI during microcatheter infusions provided essential quantification of the degree of overlap between the transcatheter perfusion territory and the enhancing mass, aiding in the selection of the faster infusion rate $(1.5 \mathrm{~mL} / \mathrm{min})$ for $12 \mathrm{~mL}$ of bevacizumab solution in the right PCA to cover a larger tumor area. The procedure time from puncture to closure was 3 hours and $50 \mathrm{~min}$, including $40 \mathrm{~min}$ in MRI.

\section{OUTCOME AND FOLLOW-UP}

No neurological deterioration was observed after the procedure except for transient visual sensations of 'moving walls' with no field cut, which resolved within 2 days. The follow-up MRI scan performed 3 days after the procedure revealed an apparent decrease in size of the enhancing mass to $32 \mathrm{~cm}^{3}$, reduced contrast enhancement, and less surrounding edema (figure 1C). A higher dose of dexamethasone (24 mg over 24 hours compared with 3-5 mg over 24 hours before treatment and at discharge) could also have contributed to the observed reduction in edema. No stroke or hemorrhage were found. The cognitive deficits improved, including an improvement in the MMSE to 25 and KPS to $70 \%$ at discharge from hospital a week later. 


\section{Key messages}

Targeted intra-arterial cerebral infusion under real-time MRI guidance is technically feasible and, while this case was performed safely, additional studies are needed to confirm a high safety profile.

- Real-time MRI is an important adjunct to X-ray based angiography as it permits visualization of transcatheter perfusion which can be overlaid with a segmented tumor area. The degree of overlap can be calculated and modifications in catheter position or infusion rate can be made to optimize targeted drug delivery to the brain.

- Intra-arterial delivery of bevacizumab might be an option for quickly declining patients with glioblastoma to rapidly improve neurological status and decrease the volume of enhancing mass, which buys time to administer other treatments.

\section{DISCUSSION}

We report the first-in-man targeted intra-arterial cerebral infusion under real-time MRI guidance, which was technically feasible and safe. The use of real-time MRI guidance provided important information that aided in the dosing of bevacizumab.

Contributors MZ: planned treatment and performed procedure, analyzed data and edited manuscript. JW: planned treatment and edited manuscript. BK: planned treatment and edited manuscript. KK: planned treatment and took care of patient on daily basis. MSP and MS: prepared virtual reality visualization. PW: planned procedure, analyzed data, edited manuscript and prepared figures. MJ: planned treatment and performed procedure, analyzed data, drafted manuscript and approved its final form.

Funding The authors have not declared a specific grant for this research from any funding agency in the public, commercial or not-for-profit sectors.

Competing interests None declared.

Patient consent for publication Not required.

Ethics approval Central Clinical Hospital of Ministry of the Interior and Administration.

Provenance and peer review Not commissioned; externally peer reviewed.

Data sharing statement All imaging data can be shared on request.

\section{REFERENCES}

1 Neuwelt EA, Frenkel EP, Diehl JT, et al. Osmotic blood-brain barrier disruption: a new means of increasing chemotherapeutic agent delivery. Trans Am Neurol Assoc 1979;104:256-60.

2 Janowski M, Walczak P, Pearl MS. Predicting and optimizing the territory of bloodbrain barrier opening by superselective intra-arterial cerebral infusion under dynamic susceptibility contrast MRI guidance. J Cereb Blood Flow Metab 2016;36:569-75.

3 Boockvar JA, Tsiouris AJ, Hofstetter CP, et al. Safety and maximum tolerated dose of superselective intraarterial cerebral infusion of bevacizumab after osmotic bloodbrain barrier disruption for recurrent malignant glioma. Clinical article. J Neurosurg 2011;114:624-32.

4 Chakraborty S, Filippi CG, Burkhardt JK, et al. Durability of single dose intra-arterial bevacizumab after blood/brain barrier disruption for recurrent glioblastoma. J Exp Ther Oncol 2016;11:261-7.

5 Alter RA, White TG, Fanous AA, et al. Long-term benefit of intra-arterial bevacizumab for recurrent glioblastoma. J Exp Ther Oncol 2017;12:67-71.

6 Lesniak WG, Chu C, Jablonska A, et al. A distinct advantage of intra-arterial delivery of intra-arterial ${ }^{89}$ Zr. J Nucl Med 2018:jnumed.118.218792. 